\title{
Mucoid Degeneration of Posterior Cruciate Ligament Leads to Roof Impingement with Anterior Cruciate Ligament: Case Report
}

\author{
Su Chan Lee, Kyung Won Choi*, Chang Hyun Nam, Seung Hyun Hwang, Hye Sun Ahn \\ Joint \& Arthritis Research, Department of Orthopaedic Surgery, Himchan Hospital, Seoul, Korea \\ Email: *evonyhair@gmail.com
}

How to cite this paper: Lee, S.C., Choi, K.W., Nam, C.H., Hwang, S.H. and Ahn, H.S. (2018) Mucoid Degeneration of Posterior Cruciate Ligament Leads to Roof Impingement with Anterior Cruciate Ligament: Case Report. Open Journal of Orthopedics, 8, 46-50.

https://doi.org/10.4236/ojo.2018.82006

Received: December 28, 2017

Accepted: February 8, 2018

Published: February 11, 2018

Copyright $\odot 2018$ by authors and Scientific Research Publishing Inc. This work is licensed under the Creative Commons Attribution International License (CC BY 4.0).

http://creativecommons.org/licenses/by/4.0/

\begin{abstract}
Mucoid degeneration of cruciate ligament is rare cause of knee pain, however there are some cases causing pain and restriction of extension. A 60-year-old man came to our clinic complaining of pain in knee joint on full range of extension about 3 months ago. The range of motion (ROM) was a flexion contracture of 5 degree and a further flexion of 140 degree with pain aggravation by forced extension. Magnetic resonance imaging of the knee joint showed thickened posterior cruciate ligament (PCL) mucoid degeneration. Arthroscopic treatment consists of PCL reduction-plasty by debridement of yellowish material in the PCL fiber and reduces the volume of the hypertrophied PCL. Immediately after surgery, patient gain the full ROM without any symptom of impingement. Enlarged PCL can make impingement in femoral notch with anterior cruciate ligament (ACL). Then, Partial debulking surgery of PCL is an effective treatment to pain relief and restore ROM of knee.
\end{abstract}

\section{Keywords}

Mucoid Degeneration, Roof Impingement, Posterior Cruciate Ligament, Anterior Cruciate Ligament

\section{Introduction}

Mucoid degeneration of anterior cruciate ligament (ACL) is a benign knee pathology with a frequency of $1.8 \%$ to $5.3 \%$ [1] [2]. Mucoid degeneration of ACL is well documented, but very little information exists about this clinical entity. This lesion of the ACL was first documented by Kumar et al. in 1999 [3]. They reported that a biopsy indicated mucoid degeneration and a relief of symptoms could be obtained through arthroscopic partial resection of the ligament. ACL 
mucoid degeneration is considered a degenerative disease characterized by diffuse infiltration of a mucoid substance in the ligament which differentiate it from cyst like lesion (synovial and mucoid cyst), and not all lesions are symptomatic [4]. ACL mucoid degeneration is more common and literature has also reported many cases, but for posterior cruciate ligament (PCL) only few cases have been reported in literature. Because unlike meniscal tears and chondral defect, reported mucoid degeneration of PCL is rare cause of knee pain. But there are some situations that make a knee pain. We herein report a case of PCL mucoid degeneration presented with secondary ACL impingement syndrome.

\section{Case Report}

A 60-year-old man came to our clinic complaining of pain in right knee joint on full range of extension about 3 months ago. The symptom appeared after physical labor using knee like walking or clean-up of his house and no significant trauma occurred during this activity. He had a partial meniscectomy for a degenerative tear of the medial meniscus 3 years before and obtained full range of motion without pain thereafter. Physical examination revealed joint effusion in his right knee with limited range of motion due to pain. The range of motion was a flexion contracture of 5 degree and a further flexion of 140 degree with pain aggravation by forced extension. No posterior sagging and knee instability was indicated by the results of anterior drawer test and pivot shift test. A plain radiograph showed tiny osteophyte and minute joint space narrowing at the medial knee joint space. Magnetic resonance imaging (MRI) of the right knee joint showed about $10 \mathrm{~mm}$ anteroposterior diameter homogenous increased signal intensity that extends longitudinally throughout a thickened PCL with an intact rim of peripheral fibers is an appearance analogues to the classic "celery stalk sign" morphology of PCL mucoid degeneration (Figure 1(a) and Figure 1(b)) [5] [6]. Medial meniscus was in partial meniscectomy state and outerbridge grade II chondromalacia on lateral side of lateral tibial spine. Based on physical examination, patient's history and characteristic of pain [7], we suspected that hypertrophic PCL with mucoid degeneration make roof impingement with ACL as the cause of the patient's symptoms.

Arthroscopic examination showed PCL was grossly bulbous thickened fibrous tissue filled with yellowish hue like ACL mucoid degeneration. On probing, tension of PCL fibers was normal and absence of PCL tear. ACL tension is normal, however, pushed toward lateral side of notch and impingement between thickened PCL and lateral wall of femoral notch during nearly full extension state (Figure 2).

Arthroscopic treatment consists of PCL reduction-plasty by debridement of yellowish material in the PCL fiber and radiofrequency ablation (RFA) proves to reduce the volume of the hypertrophied PCL. The goal was to return the PCL to its origin volume while also removing any medial superior impingement with the notch during extension and with the ACL. This volume reduction restored 


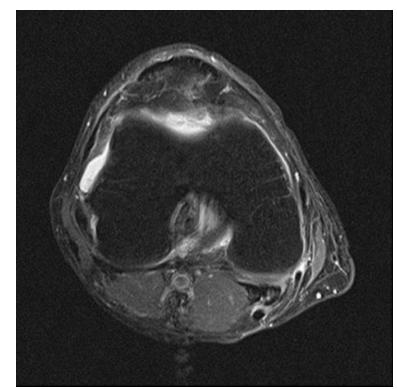

(a)

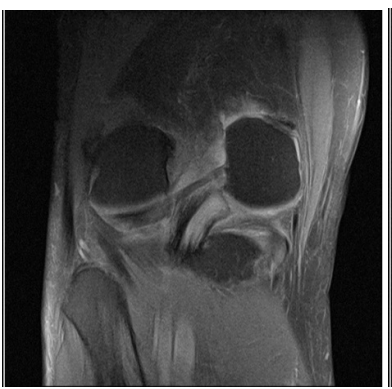

(b)

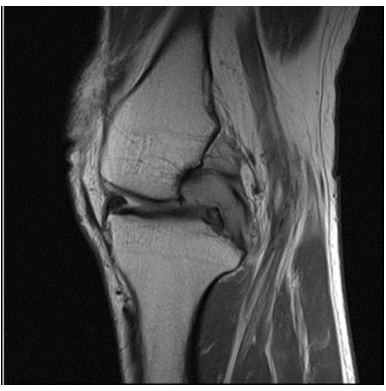

(c)

Figure 1. MRI of the knee joint. (a) Sagittal fat-suppressed T2 weighted MR image. Showing diffusely thickened and longitudinally increased signal intensity with peripheral rim of hypointense PCL fibers; (b) Coronal fat-suppressed T2 weighted MR image; (c) Axial fat-suppressed T2 weighted MR image. Notch is crowded with hypertrophic PCL and ACL.

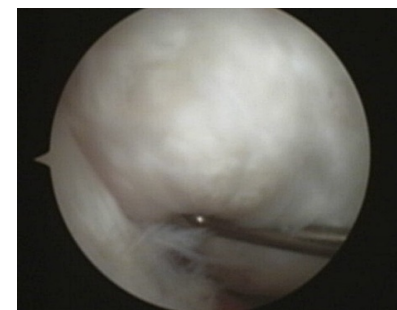

(a)

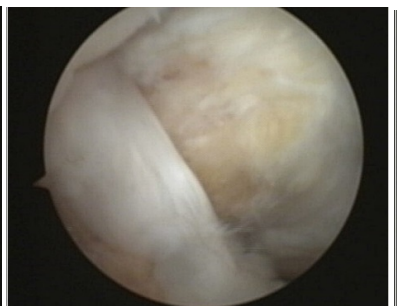

(b)

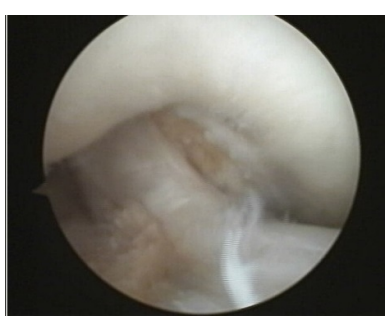

(c)

Figure 2. Arthroscopic image showing. (a) ACL impingement with grossly thickened PCL; (b) Showing the degenerative PCL and mucoid hue and filled with yellowish substance; (c) After debulking surgery, no impingement between ACL and PCL.

the space between the two cruciate ligaments and joined the pre- and retro-ligament space.

After arthroscopic procedure, Lachmann and pivot shift test were negative without instability. The patient allowed to bear weight on their leg and freely move their joint second day after the procedure. Immediately after surgery, patient gain the full range of motion without any symptom of impingement. After two-years follow up, patient remained asymptomatic. Informed consent was obtained from the patient for publication of this document.

\section{Discussion}

The true incidence of PCL mucoid degeneration is difficult to assess because it is, as in our patient population, typically asymptomatic and incidentally noted. As a point of reference, Bergin et al. reported that the incidence of ACL mucoid degeneration is $1.0 \%$ (44/4221) compare with incidence of PCL mucoid degeneration in reference was $0.1 \%(14 / 12972)$ [8]. Mucoid degeneration of PCL has been described before and there are few case reports but these cases are of asymptomatic patient or with PCL symptoms in terms of pain on flexion with restricted with terminal flexion [9]. PCL mucoid degeneration in our case presented with ACL impingement, and had restriction in terminal extension about 
5 degree, presented with pain on extension. And MRI image in sagittal plane showed appearance of PCL mucoid degeneration, like tram-track appearance [6], and in axial images notch is crowded with hypertrophic PCL and ACL (Figure 1(c)).

In arthroscopic examination, grossly thickened PCL can push an ACL towards the lateral wall and roof of femoral notch may lead to the secondary mechanical ACL impingement symptoms when terminal extension. Thickened PCL with yellowish hue are characteristic and the PCL was led with a yellowish substance. The yellowish hue was not liquid, but a fibrous tissue like ACL mucoid degeneration as described in reports on ACL mucoid degeneration [10].

We debride yellowish substance to debulk a PCL as precisely as possible to avoid posterior instability without notchplasty. Debulking of PCL hue resulted in immediate pain relief in full extension, and improved range of motion without instability. Histological examination of the biopsied tissue revealed mucoid degeneration of ligament like ACL mucoid degeneration [11]. At one year follow up, patient remains asymptomatic without instability.

\section{Conclusion}

Almost Mucoid degeneration of posterior cruciate ligament may be asymptomatic, but in case of PCL mucoid degeneration with pain and restriction in terminal extension, enlarged PCL can make impingement in femoral notch with ACL. Then, Partial debulking surgery of PCL is effective treatment to pain relief and restore range of motion of knee.

\section{Conflict of Interests}

The authors declare that they have no conflict of interest.

\section{References}

[1] Wang, J.H. and Jangir, R.R. (2015) Mucoid Degeneration of Posterior Cruciate Ligament with Secondary Impingement of Anterior Cruciate Ligament: A Rare Case Report. Journal of Orthopaedic Case Reports, 5, 44-46.

[2] Morice, A., Coupry, A., Lintz, F. and Robert, H. (2013) Reduction Plasty for Hypertrophic Anterior Cruciate Ligament Mucoid Degeneration: Clinical and Knee Laxity Outcomes in 23 Cases. Orthopaedics \& Traumatology: Surgery \& Research, 99, 693-697.

[3] Kumar, A., Bickerstaff, D.R., Grimwood, J.S. and Suvarna, S.K. (1999) Mucoid Cystic Degeneration of the Cruciate Ligament. The Journal of Bone and Joint Surgery, 81, 304-305. https://doi.org/10.1302/0301-620X.81B2.9243

[4] Parkar, A.P., Vanhoenacker, F.M. and Adriaensen, M.E. (2007) Bilateral Mucoid Degeneration of the Posterior Cruciate Ligaments. JBR-BTR, 96, 298-300.

[5] Papadopoulou, P. (2007) The Celery Stalk Sign. Radiology, 245, 916-917. https://doi.org/10.1148/radiol.2453050159

[6] McMonagle, J.S., Helms, C.A., Garrett, W.E. Jr and Vinson, E.N. (2013) Tram-Track Appearance of the Posterior Cruciate Ligament (PCL): Correlations with Mucoid Degeneration, Ligamentous Stability, and Differentiation from PCL 
Tears. American Journal of Roentgenology, 201, 394-399.

https://doi.org/10.2214/AJR.11.7400

[7] Matsumoto, A. and Howell, S.M. (2005) Avoiding Posterior Cruciate Ligament and Roof Impingement with Transtibial Anterior Cruciate Ligament Reconstruction: Keys to Correct Tunnel Placement. Techniques in Orthopaedics, 20, 211-217. https://doi.org/10.1097/01.bto.0000177270.61591.f4

[8] Bergin, D., Morrison, W.B., Carrino, J.A., Nallamshetty, S.N. and Bartolozzi, A.R. (2004) Anterior Cruciate Ligament Ganglia and Mucoid Degeneration: Coexistence and Clinical Correlation. American Journal of Roentgenology, 182, 1283-1287. https://doi.org/10.2214/ajr.182.5.1821283

[9] Okazaki, K., Deguchi, S., Katai, K. and Iwamoto, Y. (2001) Mucoid Degeneration of the Posterior Cruciate Ligament: A Case Report. Knee Surgery, Sports Traumatology, Arthroscopy, 19, 105-107. https://doi.org/10.1007/s00167-010-1171-8

[10] Cha, J.R., Lee, C.C., Cho, S.D., Youm, Y.S. and Jung, K.H. (2013) Symptomatic Mucoid Degeneration of the Anterior Cruciate Ligament. Knee Surgery, Sports Traumatology, Arthroscopy, 21, 658-663.

https://doi.org/10.1007/s00167-012-1991-9

[11] Kumar, A., Bickerstaff, D.R., Grimwood, J.S. and Suvarna, S.K. (1999) Mucoid Cystic Degeneration of the Cruciate Ligament. The Journal of Bone and Joint Surgery, 81, 304-305. https://doi.org/10.1302/0301-620X.81B2.9243 